\title{
Multi Fuels Allocation for Power Generation using Genetic Algorithm
}

\author{
Anurak Choeichum ${ }^{1, *}$,NarongdechKeeratipranon ${ }^{2}$ \\ and Chaiyaporn Khemapatapan ${ }^{3}$ \\ ${ }^{123}$ College of Innovative Technology and Engineering,Dhurakij Pundit University, \\ Bangkok, Thailand \\ earth_park@hotmail.com
}

\begin{abstract}
This paper presents solution of optimal multi fuels allocation for electric power generation planning problem via a genetic algorithm (GA). The objective is to maximize the electric power energy output and minimize the fuels cost. This is a considerably difficult problem because of its data variation.GA can provide an appropriate heuristic search method and return an actual or near optimal solution. This research used some heuristic in addition during crossover and mutation for tuning the system to obtain a better candidate solution. An experimental resultshowed a significantly improve result compared to other techniques.
\end{abstract}

Keywords:Fuels Allocation, Power Generation, Genetic Algorithm, Power Energy

\section{INTRODUCTION}

Renewable energy is widespread effectively developed throughout the globe. Many renewable energies such as wind turbine, solar farm is among the most popular alternative energy. However, the optimal management scheme of biomass fuel is not much studied. This topic is including the optimal size and location of a power plant[1], type of raw agricultural material, and the efficient process of energy conversion.Proper allocation of a raw material will have a high impact to the volume of the produced electricity. Thailand is an agricultural country andhas a plenty of biomass fuel. Currently, 2,451 MW electricity is produced from a local biomass. However,with the plenty of raw material, Thailand can produce the biomass energy at 6,500 MW maximum [2].

The optimal power generation planning has been frequently solved using classical optimization methods. The optimal power generation planning has been usually considered as the maximization of an objective function representing the energy or the minimization of an objective function representing the generation cost. The GA offer a powerful approach to these optimization problems made possible by the increasing availability of high performance computers. GAis a parallel and global search technique that emulates natural genetic operators.

GAs operates on a population of candidate solutions encoded inafinite set of bit strings called chromosome. In order to obtain optimality, each chromosome exchanges the data by operators borrowed from natural genetic to produce the better solution.

In this paper, we develop a GA applied to the problem of optimal multi fuels allocation for electric power generation planning. This research was conducted in a simulation with three different input factors; 5 type of raw materials, 4 type of energy conversion technology, and the daily pricing of electricity.

To have a high productivity, a proper match between a raw material and an energy conversion technology is required. Some raw material might suitable with one technology but not the other.This research will study to find the optimal way to match the raw material with the technology for the highest productivity along with the proper volume to suit the daily price.

\section{PROBLEM FRAMEWORKS}

This research has made preliminary structural design of the system based on the GA. Each answer is a characteristic in the form of chromosomes as shown in Fig.1,This encoding scheme is designed to 
represent the candidate solution. Starting from an arbitrary number of raw materials in the inventory, the GA process evolves the value in the chromosomes to have a good candidate solution.

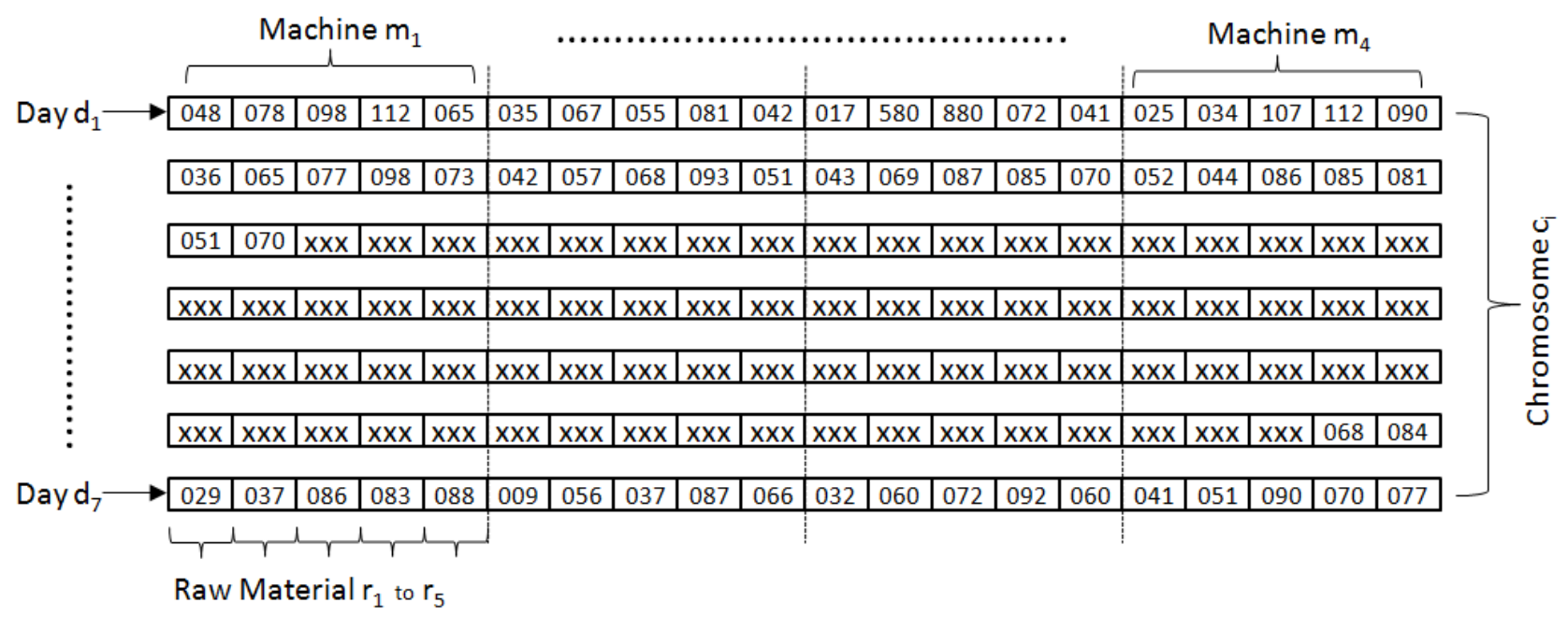

Fig.1,Conceptual illustration and components of this research

Each the chromosome of this study case has7 days with 140 genes, there are 4 machines or type of energy conversion technologies per day, and 5 type of raw materials per each energy conversion technology. Each day the daily price is $\operatorname{different}\left(P_{g}\right)$.In this study case there are the capacity factor of raw materials each type of technologies and the energy factor $\left(E_{g}\right)$ isthe energy from raw materials limitations of each technologies, as opposed to individual energy conversion technology.

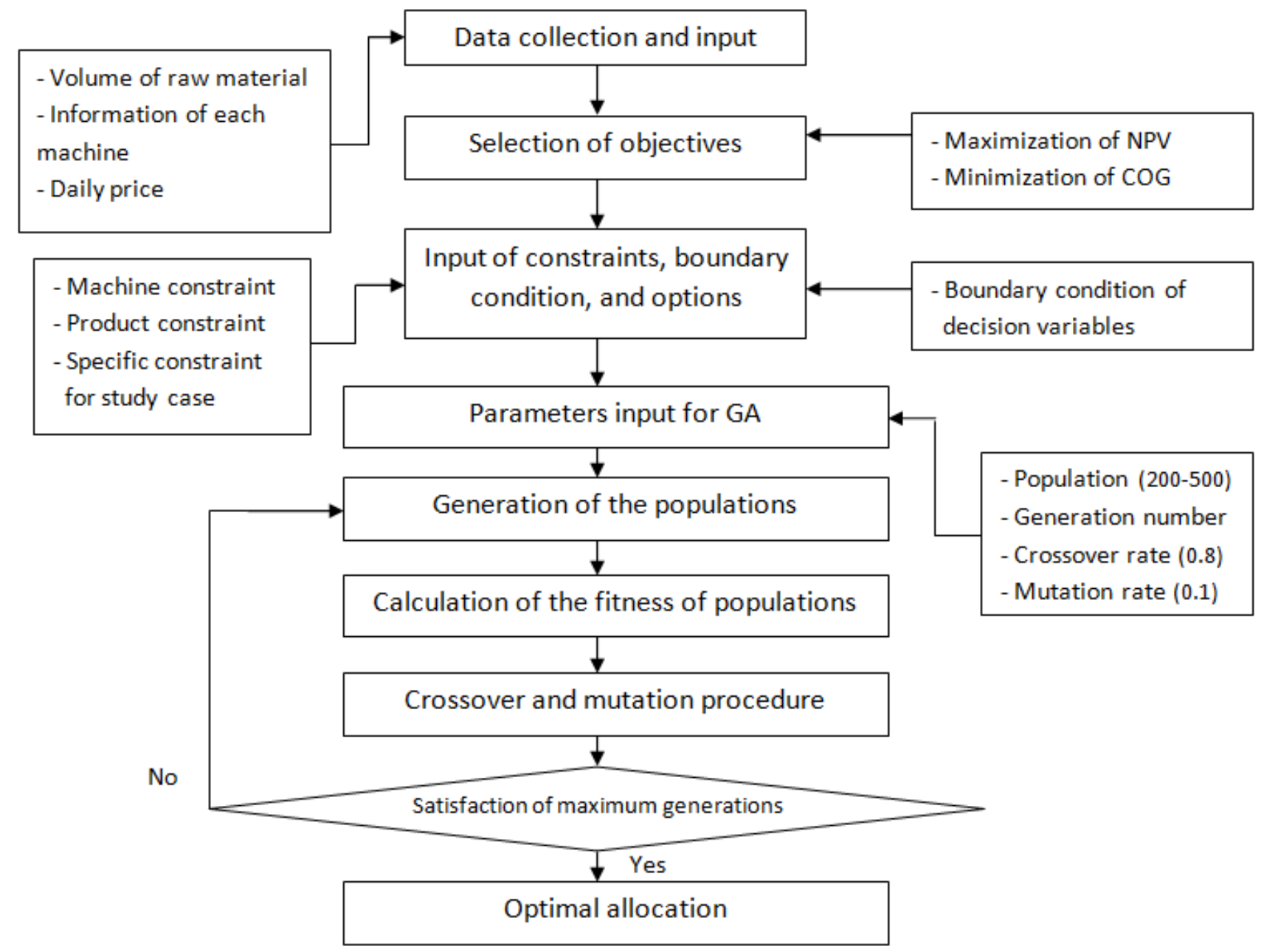

Fig.2,Flowchart of a raw material allocation using GA. 
The mathematics in thisresearch can be described by the following equations: cash inflows [3].

$$
P V_{t}=P_{g} \cdot E_{g} \cdot\left[\frac{K_{g} \cdot\left(1-K_{g V}^{u}\right)}{1-K_{g}}\right]
$$

Where:

$P V_{t} \quad$ is gained from the sold electric energy.

$P_{g} \quad$ is the selling price of electric energy.

$E_{g} \quad$ is the sold and produced electric energy.

$K_{g} \quad$ is the increase rate of sold energy price.

$V_{u} \quad$ is the useful lifetime.

\section{GENETIC ALGORITHM IN OPTIMIZE FUELS ALLOCATION}

\subsection{Chromosome and Genes}

A representation of a possible solution to an optimization problem where parameter values are encodes using either binary, real-valued or tree encoding. Genes is groups of bits or real values which encode one particular element of a possible solution (Chromosome).

Each set of chromosomes represent one individual, which is one possible solution to fuels allocation(if all constraints are satisfied then it can be considered as valid solution). Each chromosome represents four energy conversion technologies. The one energy conversion technologystores five genes in an array [4]. The index of the array specifies the position of the raw material in the energy conversion technologies.

\subsection{Crossover}

Crossover is the primary genetic operator, which promotes the exploration of new regions in the search space.An operation in which genetic material is exchanged between two different chromosomes to generate new chromosomes or child.They take information from one individual and insert it in the other to create a new child. The probability which crossover method must be used can be configured and modified $[4,5]$.

\subsection{Mutation}

Mutation is a genetic operator used to maintain genetic diversity from one generation of a population of chromosomes to the next [4]. An operation which randomly changes parts of a gene in a chromosome at randomly chosen places. The mutation adds a random search to the GA.

\subsection{Reproduction}

Reproduction is based on the principle of better fitness survival. It is an operator that obtains a fixed copies number of solutions according to their fitness value. If the score increase, the number of copies increases too. A score value is associated to a solution related on its distance from the optimal solution [5].

\subsection{Fitness}

To choose which children from the newly created population will be favored to breed, the fitness of every individual has to be computed. In this study case, the Eq.3, is the fitness function of this implementation.

$$
\begin{gathered}
\max \sum_{t=1}^{T} P_{t} \\
P_{t}=P V_{t}-\left[I n v_{s}+L P_{t}+M C_{t}\right](3)
\end{gathered}
$$

Where:

$P_{t}$ is the maximum of selling.

$\operatorname{Inv} v_{S}$ is the investment cost in the production cycle.

$L P_{t} \quad$ is the electricity used in manufacturing.

$M C_{t} \quad$ is the maintenance technology. 


\subsection{Termination of the GA}

Because GA is a stochastic search method, it is difficult to formally specify convergence criteria. As the fitness of a population may remain static for a number of generations before a superior individual is found, the application of convergence termination criteria becomes problematic, a common practice is to terminate GA after a specified number of generationsand then test the fitness of best members in the last population. If no acceptable solutions are found, the GA may be restarted or fresh search initiated [5-8].

3.7 Selection process depending on the Fitness

The calculated fitness helps now to select members for the next generation. This is done using the Roulette Wheel Selection method where individuals with a higher fitness are more likely to be selected then others [4-8].

\section{RESULTS}

The results in Fig. 3, shows the 200 population size compared with 500 population size.From this experiment, a GA with higher population size can find the best answer using lower iteration. In the Fig. 4, shows each mutation technique can descript that the number of mutation $\mathrm{T}$ and mutation $\mathrm{S}$ is the population size $\left(' 1^{\prime}=200,{ }^{\prime}{ }^{\prime}=500\right)$ and the $\mathrm{T}$ is the probability ratio of mutation 10 percentage, and $\mathrm{S}$ is the probability ratio of mutation 5 percentage $(\mathrm{T}=10 \%, \mathrm{~S}=5 \%)$.
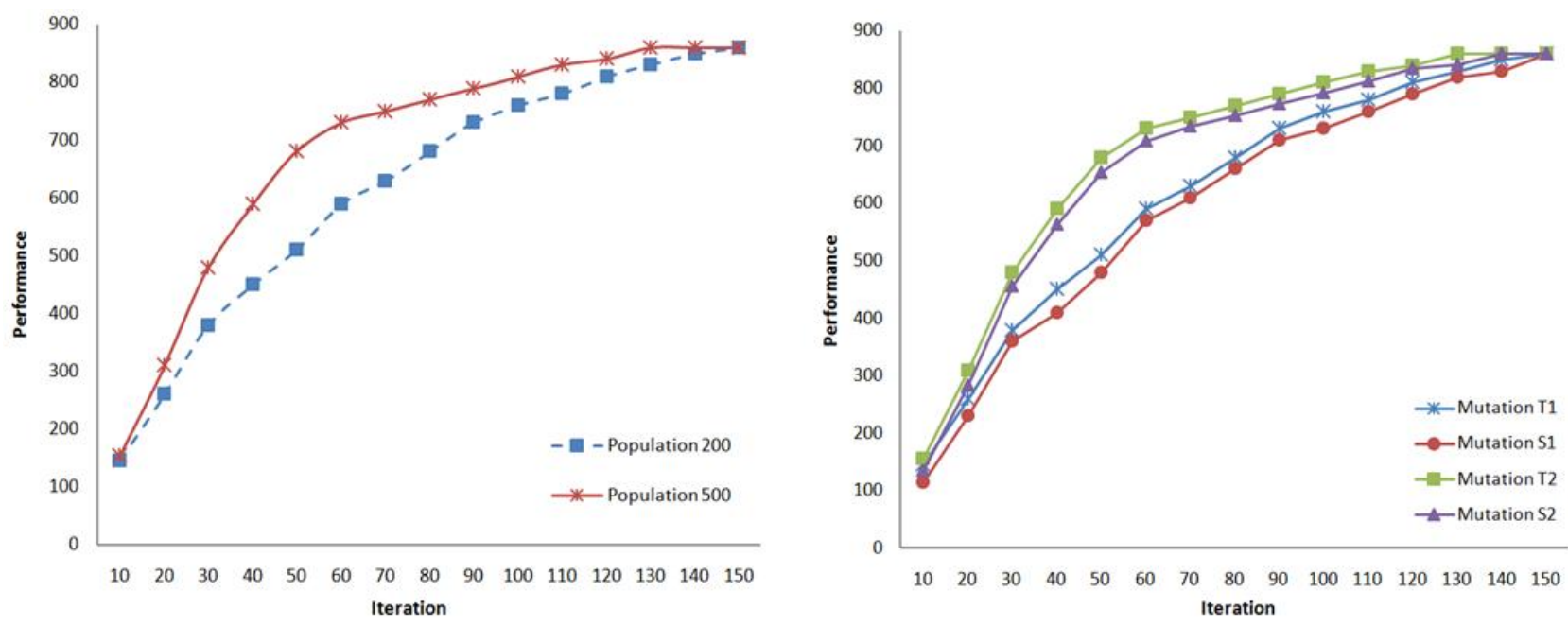

Fig.3,Comparison of each population size.Fig.4,Comparison of each mutation technique.

\section{DISCUSSIONS}

From the result, the numbers of population has inverse proportion to the required iteration. This is because the bigger volume of the population can add more chance to find a good candidate solution. In the mutation operation, each gene or quantity of each raw material has a probability to get a mutation. If a gene is selected for mutation, the new value of the gene will be a random value within the range of $+/-100 \%$ from its previous value. In this case, a higher rate of mutation can lead to a quicker solution because its population try a new value more often.

\section{CONCLUSIONS}

This research was conducted using genetic algorithm to allocate multi fuels for power generation planning for the best solution. This research successfully demonstrates a method to provide the suitable raw material allocation when there are a different in raw material, energy conversion technologies, and daily tariff rate. With this system, an investor can have a better allocation plan and hence receive more electricity output from the plant. In response to the government power 
development plan, this project can provide a solution for a stability to the power grid, optimize energy conversion and help to satisfy decision-makers' requirements for maximum investment returns.

\section{References}

[1] M. Sedighizadeh M. Rafiei A. Hakimi, "OPTIMIZING A TYPICAL BIOMASS FUELED POWER PLANT USING GENETIC ALGORITHM AND BINARY PARTICLE SWARM OPTIMIZATION", International Journal on "Technical and Physical Problems of Engineering" (IJTPE), June 2013, Issue 15, Vol 5, Pg 15-21.

[2] Alternative Energy Development Plan: AEDP2015, Department of Renewable Energy Development and Energy Efficiency, September 2015

[3] MojtabaRafiei, MostafaSedighiZadeh"Optimization of a typical biomass fueled power plant using Genetic Algorithm and Binary particle swarm optimization", IEEE

[4] Abdul Kadar Muhammad Masum, Mohammad Shahjalal,"Solving the Vehicle Routing Problem using Genetic Algorithm", (IJACSA) International Journal of Advanced Computer Science and Applications, Vol. 2, No. 7, 2011

[5] M. YOUNES, M. RAHLI AND L. ABDELHAKEM-KORIDAK,"Optimal Power Flow Based on Hybrid Genetic Algorithm", JOURNAL OF INFORMATION SCIENCE AND ENGINEERING 23, 1801-1816 (2007)

[6] A. Arabali, M. Ghofrani, M. Etezadi-Amoli, M. S. Fadali and MoeinMoeini-Aghtaie, "A Multi-Objective Transmission Expansion Planning Framework in Deregulated Power Systems With Wind Generation", IEEE TRANSACTION ON POWER SYSTEMS, VOL.29, NO 6, NOVEMBER 2014

[7] T. Senjyu, D. Hayashi, N. Urasaki and T. Funabashi, "Optimum Configuration for Renewable Generating Systems in Residence Using Genetic Algorithm”, IEEE TRANSACTIONS ON ENERGY CONVERSION, VOL. 21, NO.2, JUNE 2006

[8] Warsono, D. J. King, C. S. Özveren and D.A. Bradley, "Economic Load Dispatch Optimization of Renewable Energy in Power System Using Genetic Algorithm", PowerTech, IEEE, 2007, Page 2174-2179 